\title{
Targeting vascular endothelial growth factor using retinal gene therapy
}

\author{
Sook H. Chung, Sonia L. Frick, Glenn Yiu \\ Department of Ophthalmology \& Vision Science, University of California, Davis, Sacramento, CA, USA \\ Contributions: (I) Conception and design: SH Chung, G Yiu; (II) Administrative support: G Yiu; (III) Provision of study materials or patients: None; (IV) \\ Collection and assembly of data: SH Chung, SL Frick; (V) Data analysis and interpretation: SH Chung, G Yiu; (VI) Manuscript writing: All authors; \\ (VII) Final approval of manuscript: All authors. \\ Correspondence to: Glenn Yiu, MD, PhD. 4860 Y St., Suite 2400, Sacramento, CA 95817, USA. Email: gyiu@ucdavis.edu.
}

\begin{abstract}
Pharmacotherapies targeting vascular endothelial growth factor (VEGF) have revolutionized the management for neovascular retinal disorders including diabetic retinopathy and neovascular age-related macular degeneration. However, the burden of frequent injections, high cost, and treatment resistance in some patients remain unresolved. To overcome these challenges, newer generations of anti-angiogenic biological therapies, engineered proteins, implantable delivery systems, and biopolymers are currently being developed to enable more sustained, longer-lasting treatments. The use of gene therapies for pathologic angiogenesis has garnered renewed interests since the first FDA-approval of a gene therapy to treat inherited retinal diseases associated with biallelic RPE65 mutations. Newer generations of viral vectors and novel methods of intraocular injections helped overcome ocular barriers, improving the efficiency of transduction as well as safety profile. In addition, unlike current anti-VEGF gene therapy strategies which employ a biofactory approach to mimic existing pharmacotherapies, novel genome editing strategies that target proangiogenic factors at the DNA level offer a unique and distinct mechanistic approach that can potentially be more precise and lead to a permanent cure. Here, we review current anti-VEGF therapies and newer pharmacologic agents under development, examine technologies and progress in adapting anti-VEGF gene therapies, and explore the future application of CRISPR-Cas9 technology to suppress ocular angiogenesis.
\end{abstract}

Keywords: Angiogenesis; vascular endothelial growth factor (VEGF); gene therapy; neovascular age-related macular degeneration (nAMD); neovascularization

Submitted Jun 01, 2020. Accepted for publication Sep 25, 2020.

doi: $10.21037 /$ atm-20-4417

View this article at: https://dx.doi.org/10.21037/atm-20-4417

Vascular endothelial growth factor (VEGF) is a proangiogenic cytokine that has been implicated in a variety of retinal disorders including diabetic retinopathy, retinal vein occlusion (RVO), and neovascular age-related macular degeneration (nAMD). It is encoded in 8 exons located on chromosome 6 , and its functions include stimulating endothelial cell migration, proliferation and tube formation, all of which lead to neovascularization in the eye (1). The VEGF family consists of 7 members (VEGFa-VEGFe and placental growth factor or PGF), among which the secreted isoforms of VEGFa (VEGF121 and VEGF165) are the most potent factors associated with pathologic angiogenesis $(1,2)$. VEGF is primarily an endothelial cell mitogen, but may also be secreted by retinal pigmented epithelium (RPE), Muller glia, and astrocytes (3-6). Early success in pre-clinical studies using intravitreal injections of anti-VEGF antibody in the 1990s led to clinical trials and subsequent approval for human use, enabling anti-VEGF therapies to become the standard of care for various ocular angiogenic disorders (7-10). The challenges with current anti-VEGF pharmacotherapies, however, include short durability requiring frequent injections, limited efficacy in some patients, as well as infection risk and high costs of multiple treatments. In this review, we review current anti- 
Table 1 Summary of Anti-VEGF drugs

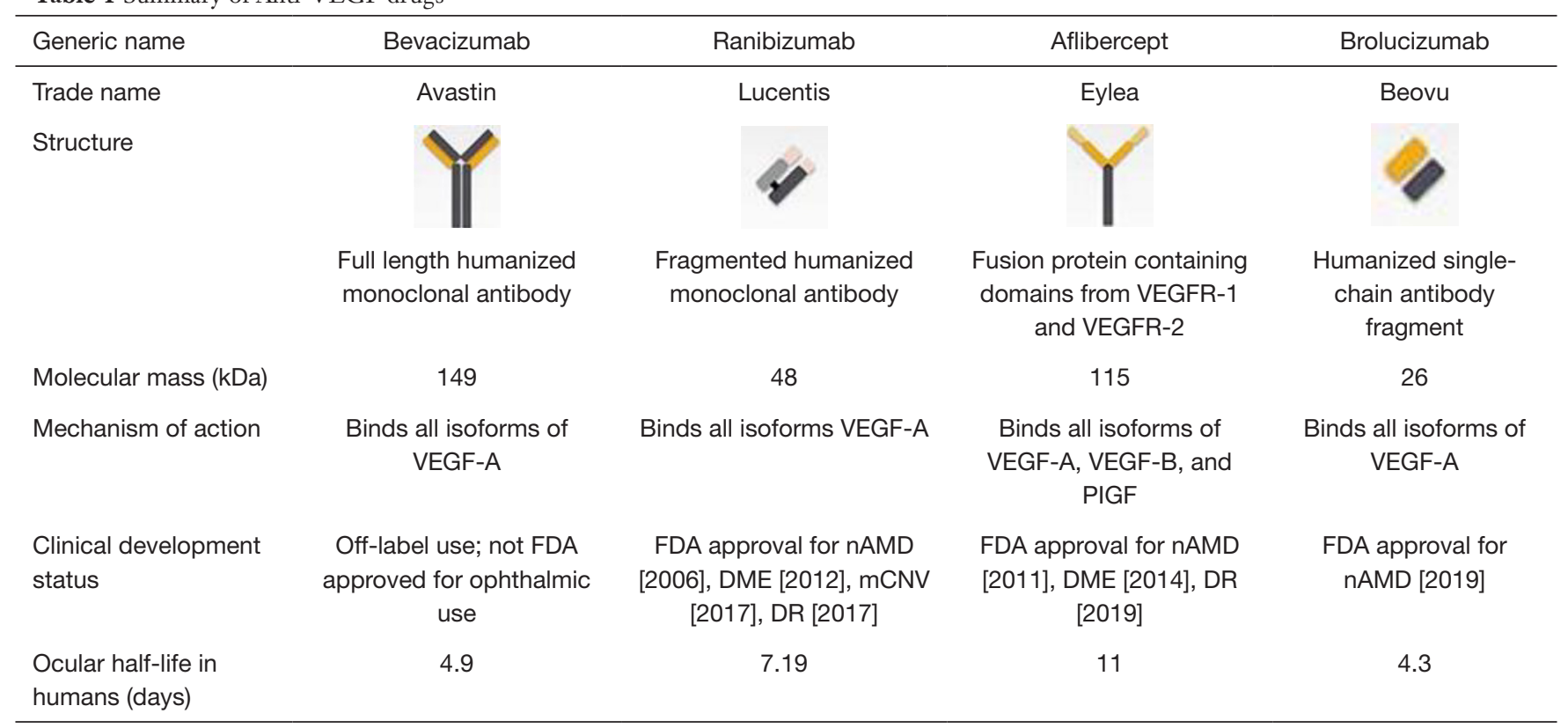

VEGF, vascular endothelial growth factor; PIGF, placental growth factor; nAMD, neovascular age related macular degeneration; DME, diabetic macular edema; mCNV, myopic choroidal neovascularization; DR, diabetic retinopathy.

VEGF treatments and existing strategies to improve drug efficacy and durability, then discuss the use of gene therapy and potential applications of genome editing technology for treatment of neovascular retinal conditions.

\section{Current anti-angiogenic therapies for retinal diseases}

Prior to the advent of pharmacologic interventions, the primary mode of inhibiting retinal angiogenesis was with thermal laser. Eyes with proliferative diabetic retinopathy could be treated with pan retinal photocoagulation, while choroidal neovascularization (CNV) in NAMD could be treated with laser ablation if the fovea were spared, then later with photodynamic therapy (PDT) using a porphyrinbased photosensitizer (verteporfin) if the fovea were involved. These destructive laser treatments were designed to halt the disease progression but can cause permanent damage of target retina and subsequent vision loss. In the early 2000s, intravitreal injections of agents targeting VEGF, known previously as "factor X", led to a fundamental paradigm shift in the management of neovascular retinal conditions, enabling regression of aberrant neovessels. Above, we summarize the current generation of anti-VEGF pharmacotherapies (Table 1).

\section{Bevacizumab}

Bevacizumab is a humanized full-length VEGF monoclonal antibody $(149 \mathrm{kDa})$ that binds to all isoforms of VEGFa (11). Initially approved for metastatic colon cancer, bevacizumab given systemically demonstrated benefit in patients with nAMD, enabling not only a reduction in $\mathrm{CNV}$ and macular thickness, but also significant improvement in visual acuity (12). When given intravitreally off-label, the treatment continued to show significant anatomic and functional benefits while avoiding systemic side effects (13). Although it never received approval by the Food and Drug Administration (FDA), bevacizumab remains one of the most commonly used treatments due to its clinical benefits and lower cost compared to approved therapies. A randomized controlled trial directly comparing $1.25 \mathrm{mg}$ bevacizumab with the FDA-approved ranibizumab, which is many times more expensive, demonstrated similar benefits, supporting the use of bevacizumab as first-line therapy in most clinical scenarios (14).

\section{Ranibizumab}

Ranibizumab is a humanized monoclonal antibody fragment similar to the binding (Fab) region of bevacizumab that was developed specifically for intraocular use (15). The smaller 
size of ranibizumab (48 $\mathrm{kDa}$ ) compared to bevacizumab (149 $\mathrm{kDa})$ was designed to enable better penetration through the neurosensory retina and reduce systemic exposure or potential adverse effects, making it safer for ophthalmic use (16). Its efficacy was demonstrated in two phase III clinical trials (MARINA, NCT02147067 and ANCHOR, NCT00061594) which demonstrated efficacy for treating different types of $\mathrm{CNV}$ in $\mathrm{nAMD}$ as compared with observation or PDT $(17,18)$, resulting in its approval for nAMD in 2006. The drug was subsequently found to benefit eyes with RVO-related macular edema in the BRAVO (NCT00486018) and CRUISE (NCT00485836) studies $(19,20)$, and eyes with diabetic macular edema in the RIDE (NCT00473382) and RISE (NCT00473330) trials (21), resulting in its approval for these two conditions in 2010 and 2012, respectively. Due to small potential safety concern in cardiovascular endpoints, ranibizumab was approved at $0.3 \mathrm{mg}$ for diabetic patients, compared to the $0.5 \mathrm{mg}$ dose for nAMD and RVO patients. More recently, ranibizumab has also been approved for the treatment of myopic $\mathrm{CNV}$ as well as diabetic retinopathy in 2017.

\section{Aflibercept}

Aflibercept is a recombinant chimeric fusion protein that consists of key domains of human VEGF receptors 1 and 2 with the constant region (Fc) of human $\operatorname{IgG}(14,22)$. It functions as a decoy receptor that can bind to VEGFa and PGF and hinder their functions (23). Its higher binding affinity and longer half-life in the vitreous was believed to improve the efficacy and durability of the drug (24). The phase III VIEW 1 and 2 trials (NCT00509795 and NCT00637377) demonstrated non-inferiority of aflibercept given every 8 weeks as compared to ranibizumab given monthly for nAMD, although an equivalent head-tohead comparison was not conducted $(22,25)$. Based on this data, 2-mg aflibercept was FDA-approved for nAMD in 2011. Subsequent completion of the COPERNICUS (NCT00943072), GALILEO (NCT01012973), and VIBRANT (NCT01521559) trials, as well as the VIVID (NCT01331681) and VISTA (NCT02299336) studies (26-29), led to the drug's approval for RVO-related and diabetic macular edema in 2014. Following ranibizumab, aflibercept was also later approved for diabetic retinopathy in 2019. Despite the presumed greater efficacy and durability of aflibercept, long-term studies have not shown a difference in visual benefits (30).

\section{Brolucizumab}

Brolucizumab is a single-chain antibody fragment targeting VEGFa, which due to its much smaller size (23 kDa), allows a much higher dose to be delivered intravitreally $(31,32)$. A comparison between brolucizumab and other antiVEGF drugs suggests that it has similar or superior binding affinity to human VEGF (33), and showed early promise in phase II studies $(34,35)$. The HAWK (NCT02307682) and HARRIER (NCT02434328) phase III trials found that over $50 \%$ of the patients that received intravitreal injections of 6-mg brolucizumab were able to maintain 3-month intervals for additional treatment through week 48 suggesting improved durability, resulting in the drug's approval by the FDA in 2019. Some reports of an obstructive vasculitis distinct from the mild intraocular inflammation typically seen in early deployment of other anti-VEGF therapies have been reported after brolucizumab treatment, raising some concerns (36). Further studies are required to confirm the long-term safety of this treatment.

\section{Emerging anti-angiogenic therapies}

Despite the successes of current anti-VEGF therapies, their limited durability and treatment resistance in subsets of patients remain areas of unmet needs. Frequent injections increase the risk of endophthalmitis, retinal detachment, elevated ocular pressure, and vitreous hemorrhage (37). In particular, endophthalmitis is associated with poor visual prognosis $(38,39)$. Thus, new technologies aimed at greater efficacy and durability are under development, as summarized below (Table 2).

\section{Port delivery system (PDS)}

The PDS is a refillable, non-biodegradable implant that enables continuous delivery of drug to the vitreous (40). It can be permanently inserted via a small incision through the sclera with a self-sealing valve, and releases drugs into the vitreous through passive diffusion. A phase II clinical trial delivering ranibizumab through the PDS demonstrated safety and a median refill time of 15 months (NCT02510794), suggesting that sustained VEGF suppression may be therapeutic at lower doses than the pulsatile dosing from multiple intravitreal injections (40). Although a high rate of vitreous hemorrhage at the incision site was noted in earlier stages of the trial, the surgical procedure has been optimized and hemorrhage occurrence 


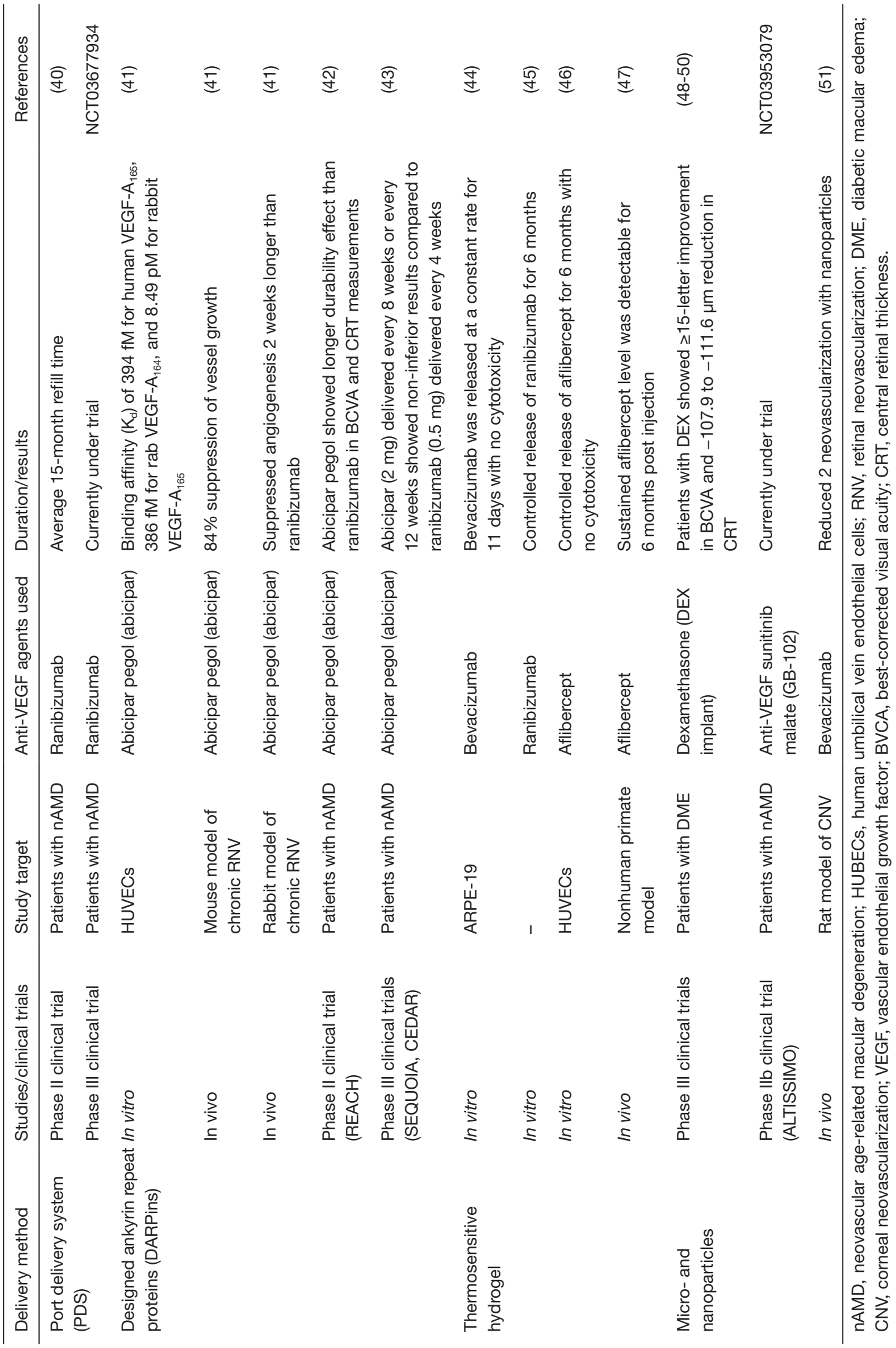


has decreased (40). A phase III trial assessing its efficacy and pharmacokinetics is currently underway in patients with nAMD (NCT03677934).

\section{Designed ankyrin repeat proteins (DARPins)}

DARPins are genetically engineered proteins that mimic antibodies with equal or superior binding affinity and specificity (52). They are smaller in size, enabling better penetration with a longer half-life ( $>13$ days) than ranibizumab (7.2 days) (53-55). For ophthalmic applications, abicipar pegol, an anti-VEGF DARPin, demonstrated effective suppression of vascular leak and neovascularization in animal models of corneal neovascularization and retinal vasculopathy (41). Phase III clinical trials (SEQUOIA (NCT02462486) and CEDAR (NCT02462928)] evaluating abicipar pegol given every $2-3$ months in nAMD patients demonstrated non-inferior in improving visual acuity compared with monthly treatment of ranibizumab (41-43).

\section{Thermosensitive hydrogel}

Hydrogels are three-dimensional networks of hydrophilic polymers that can hold large quantity of water content similar to natural tissue $(56,57)$. Thermosensitive hydrogels are liquid at room temperature, but becomes solid at body temperature, allowing the drug-carrying polymer to be injected intravitreally, but serve as a reservoir for slow, sustained drug delivery inside the eye $(58,59)$. For ophthalmic use, Wang et al. evaluated a biocompatible material composed of an amphiphilic triblock of copolymer of poly(2-ethyl-2-oxazoline)-b-poly(e-caprolactone)b-poly(2-ethyl-2-oxazoline) (PEOz-PCL-PEOz) and demonstrated extended release of bevacizumab with no significant toxicity in the rabbit retina (44). More recently, hydrogels also demonstrated controlled release of ranibizumab and aflibercept in vitro $(45,46)$, and when injected intravitreally showed sustained release of aflibercept over 6 months in nonhuman primates without significant adverse effects (47).

\section{Micro- and nanoparticles}

Biodegradable microparticles and nanoparticles also hold great potential for sustained delivery of drugs. The use of engineered polymeric microparticles of poly lacticco-glycolic acid (PLGA) was previously approved by the
FDA for inflammatory diseases. For ocular delivery, the microparticles are designed to aggregate upon exposure to vitreous fluid to form a depot at the bottom of the eye to gradually release the loaded compound. The PLGAbased intravitreal implant for dexamethasone has been used successfully in patients with diabetic macular edema with good efficacy (Ozurdex, AbbVie-Allergan) (48-50). The ongoing ALTISSIMO phase IIb trial (NCT03953079) is designed to evaluate PLGA microparticles carrying the anti-VEGF sunitinib malate (GB-102) in eyes with diabetic macular edema. Similar to microparticles, nanoparticles are also synthetic polymeric drug carriers but are nanometer in size. Nanoparticles have mainly been tested in preclinical studies in rats and mice, and showed promise suppressing ocular angiogenesis by topical application $(51,60)$.

\section{Gene therapy considerations for neovascular retinal diseases}

Gene therapy has several advantages over pharmacological treatments including long-term therapeutic effects without repeated treatments, and the capacity for cell-targeted delivery using cell-specific promotors. There has been tremendous excitement surrounding the first FDA-approval of a retinal gene therapy for retinal degenerations associated with biallelic loss of the RPE65 gene $(61,62)$. However, unlike most inherited retinal diseases which are caused by single gene mutations, retinal angiogenesis involves a complex network of many different pro-angiogenic and anti-angiogenic factors. Also, while no current therapies are available for most inherited retinal degenerations, many treatments already exist for neovascular retinal diseases. While gene therapies for inherited conditions target younger patients before the onset of blindness, degenerative conditions such as AMD primarily impact older adults, where the quality-adjusted life-year gain may not necessarily justify the cost and risks of a new therapy. Nevertheless, given the tremendous burden of pharmacologic anti-VEGF treatments, gene therapy holds the promise for long-term suppression of VEGF in neovascular retinal diseases.

Gene therapy involves the delivery of a therapeutic gene into retinal cells using a gene-carrying vector. However, the specific vector and mode of delivery depends on the choice of the therapeutic gene, target cell type, and target region of transduction. Here we discuss gene delivery vectors and modes of intraocular delivery in designing a gene therapy strategy for neovascular retinal diseases. 
Table 3 Types of transgene delivery vehicles for ocular gene therapy

\begin{tabular}{lcccc}
\hline Vehicle property & Lentivirus & Adenovirus & AAV & Synthetic \\
\hline Expression level & High & High & High & Long-term \\
Expression duration & Long-term & Transient & $\sim 5 \mathrm{~kb}$ & Variable \\
Packaging capacity & $\sim 8 \mathrm{~kb}$ & $\sim 9 \mathrm{~kb}$ & Low & Variable \\
Immunogenicity & High & High & Low & Low \\
Risk of mutation & High & Low & Low \\
\hline
\end{tabular}

$\mathrm{AAV}$, adeno-associated virus.

\section{Gene delivery vectors}

The most common carriers for delivering genetic material are viral vectors. Although synthetic polymers such as PLGA nanoparticles have been extensively evaluated as gene-carrying vectors, and are generally safe with low immunogenicity (63), these non-viral carriers are generally less efficient at transducing retinal cells compared with viral vectors. Common viral delivery platforms include lentivirus, adenovirus, and adeno-associated virus (AAV), each of which has distinct advantages and disadvantages (Table 3).

\section{Lentiviral vectors}

Lentiviral vectors are single-stranded RNA viruses that can deliver $\sim 8$-kb long transgene to both dividing and nondividing cells. They integrate into the host genome to enable sustained and long-lasting transgene expression, but carries a risk of mutagenesis if it integrates into a tumor suppressor gene. To overcome this issue, inactivation at the 3' long terminal repeat (LTR) has been developed for selfinactivation vectors (64).

\section{Adenoviruses}

Adenoviruses are double-stranded DNA viruses that can package larger genes $(9 \mathrm{~kb})$, but its infectivity is limited to postmitotic cells. While adenoviruses can efficiently infect a variety of retinal cell types, they have largely been abandoned due to significant host immune responses which results in a loss of therapeutic effect.

\section{Adeno-associated virus}

AAV vectors are the leading platform for in vivo gene delivery as they have been engineered to exclude intrinsic viral sequences resulting in low immunogenicity and cytotoxicity (65). Despite its limited genome packaging size $(\sim 4.7 \mathrm{~kb}), \mathrm{AAV}$ has been widely-used both in preclinical and clinical studies as recombinant vectors with pseudo-typed capsids that can achieve cell-specific therapy. For example, retinal ganglion neurons can be infected with AAV2 and AAV8 given intravitreally, while photoreceptors and RPE cells can be efficiently transduced with AAV2, AAV5, AAV7, AAV8, and AAV9 after subretinal delivery (66-68). Typically, $\mathrm{AAV}$ vectors in the vitreous cavity cannot transduce photoreceptors or RPE due to the internal limiting membrane (ILM) barrier that is formed by the foot plates of Muller glia. Using a method of "directed evolution" where libraries of AAV variants are rapidly screened in vivo for cell-type tropism and transduction efficiency, newer generations of AAV such as the AAV2-7m8 vector have been developed to overcome the ILM barrier to transduce outer retinal neurons after intravitreal injection (69).

\section{Modes of vector delivery}

Intraocular delivery of viral vectors enables localized transduction of different retinal cell types with minimal systemic exposure. Intraocular injections require a smaller amount of virus to be delivered than intravenous delivery, and due to the immune privileged status of the eye, can limit host immune responses that could otherwise cause cellular damage or reduce transduction efficiency. However, different modes of intraocular delivery vary with regard to ease of application, biodistribution, and immunogenicity. Here, we discuss 3 major modes of vector delivery (Figure 1).

Subretinal injections are the most common route of viral delivery in the eye because it readily bypasses the ILM barrier and produces reliable, robust transgene expression in outer retinal cells that are the target of most gene therapy strategies. Because the viral particles are confined to the immune-privileged subretinal space, host immune responses are minimal, and the degree of intraocular inflammation is very mild. However, subretinal injections are performed using a transretinal cannula that must be inserted through 


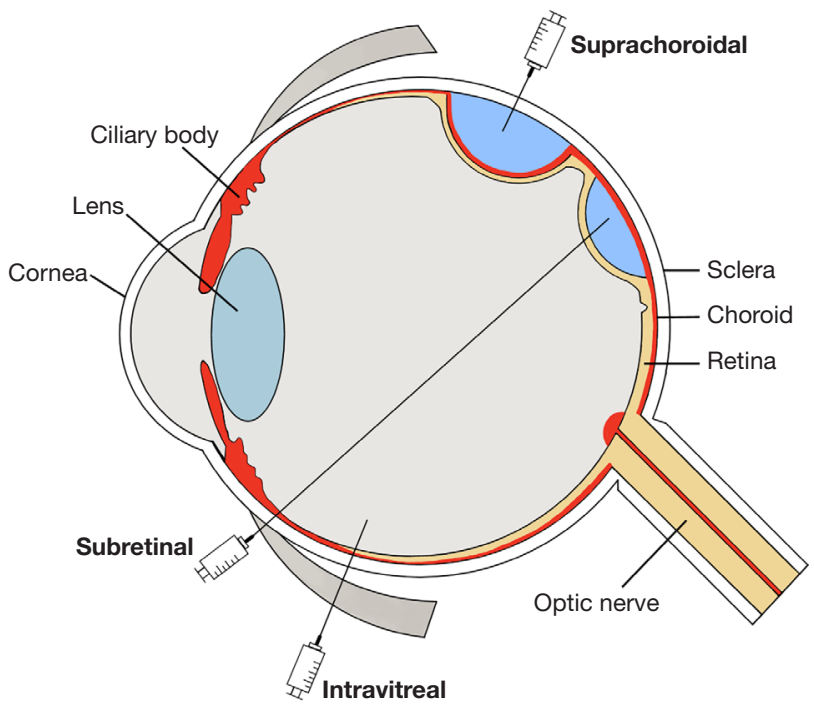

Figure 1 A schematic diagram illustrating 3 different intraocular injection methods; subretinal, intravitreal and suprachoroidal injections.

the neurosensory retina to create a retinotomy-a technique that generally requires complex vitrectomy surgery and has a risk of retinal detachment. Moreover, the transduction area is limited to the small area created by the injected fluid bleb, so widespread expression is difficult to achieve. The first FDA-approved ocular gene therapy for RPE65 employs subretinal AAV2 delivery, and has demonstrated both safety and efficacy in clinical trials.

Intravitreal injections are commonly performed by retinal specialist for delivering pharmacotherapies such as steroids and anti-VEGF agents. These injections are easy to perform in an office setting, have low risks of endophthalmitis or retinal detachment, and can be repeatedly given. Unlike subretinal injections, the injected viral particles can distribute broadly to transduce the entire retina, rather than just a small region. Although efficient transduction of outer retinal layers is limited by the ILM barrier, surgical removal of ILM may improve transduction efficiency, although it still requires intraocular surgery (70). Newer generations of AAV such as AAV2-7m8 and some tyrosine mutants have been shown to exhibit better penetration and transduction efficiency when given intravitreally (69). However, intravitreal viral injections generally cause more intraocular inflammation than subretinal delivery, possibly due to the greater degree of trabecular outflow to the systemic circulation as compared to uveoscleral outflow.
Suprachoroidal injections are a novel mode of intraocular delivery that uses microneedles or microcatheters to access a potential space between the choroid and the scleral wall of the eye (71-73). Suprachoroidal delivery of triamcinolone are effective in treating macular edema resulting from RVO and uveitis $(74,75)$. Recent studies of suprachoroidal AAV delivery demonstrated widespread transgene expression and greater vector coverage in outer retinal cells, although mostly confined to the peripheral retina $(76,77)$. Compared to methods using microcatheters $(78,79)$, custom microneedles can be used in office settings similar to intravitreal injections (77). However, because the suprachoroidal space is outside the blood-retinal barrier delimiting the zone of immune privilege, there is a higher potential risk of inflammation or host immune responses.

The choice of viral vector and delivery mode depends significantly on the choice of the therapeutic transgene, the target cell type, and target region of transduction. For example, for gene replacement strategies, the cell types that natively produce the mutated gene product should at least be transduced. However, if the transgene is a secreted protein such as an anti-VEGF antibody or decoy VEGF receptor, the identity of the transduced cells may not be important, as they essentially serve as a "biofactory". For diseases such as nAMD, the treatment effect only needs to be localized to the area of the $\mathrm{CNV}$, while global ischemic conditions such as proliferative diabetic retinopathy may require broader areas of therapeutic effect. However, if the therapeutic transgene can exert its effect at a distance, it may be more beneficial to transduce cells farther away from the pathologic region or macula area to minimize any potential damage from the injection procedure itself. Since anti-angiogenesis gene therapy strategies vary widely, we review several approaches most actively under investigation.

\section{Anti-VEGF gene therapy strategies}

\section{$r A A V-s F l t 1$}

One of the first anti-VEGF gene therapy strategies employed subretinal injections of recombinant AAV2 vector expressing soluble VEGF receptor 1 , sFlt-1. Several pre-clinical studies using a transgenic mouse model and non-human primates have shown that a single subretinal injection of rAAV-sFlt1 was well tolerated, and suppressed angiogenesis effectively without significant adverse effects or host immune responses (80-82). However, although phase I studies (NCT01494805) in nAMD patients demonstrated 
safety (83), the phase IIa randomized clinical trial with 32 patients showed no clear benefit in visual acuity or anatomy compared with baseline and control eyes (84). Another phase I clinical trial with AAV2-sFlt01 (Sanofi Genzyme) was conducted (NCT01024998) with 19 nAMD patients, which proved its safety, but showed high variability in sFlt expression and anti-permeability between patients (85). Interestingly, 5 of 10 patients did not express sFlt01 after receiving AAV2-sFlt01 $\left(2 \times 10^{10} \mathrm{vg}\right)$, and 4 of these 5 nonexpressors had serum neutralizing antibody titer greater than 1:400 (85), suggesting that humoral immune responses impacted efficacy. These small early studies enrolled patients with chronic nAMD who received previous antiVEGF injections or showed minimal response to antiVEGF agents at baseline. Thus, the potential effectiveness of gene therapy strategies for neovascular retinal conditions was not fully evaluated.

\section{ADVM-022}

To overcome the difficulties of subretinal injections, ADVM-022 employs the AAV2-7m8 vector to encode aflibercept to be given as an intravitreal injection. A preclinical study with laser-induced CNV in non-human primates found ADVM-022 effective at maintaining high aflibercept levels in the vitreous for 3-9 months with no serious adverse effects (86), and prevented laser-induced $\mathrm{CNV}$ at levels comparable to a single intravitreal aflibercept at the time of CNV induction (86). The multicenter, openlabel, dose-ranging phase I clinical trial (OPTIC trial, NCT03748784) is currently ongoing, and early reports found 10 out of 12 patients did not require a rescue injection for 24 weeks while maintaining visual acuity and reduction in central retinal thickness (87). A more recent report showed that the 6 patients who received the higher $6 \times 10^{11} \mathrm{vg}$ dose of ADVM-022 maintained vision and anatomy on optical coherence tomography (OCT) without rescue injections through a median of 34 weeks (range, 24-44 weeks) (88).

\section{$R G X-314$}

RGX-314 is an AAV8 vector that expresses an antiVEGF antibody fragment (Fab) that selectively binds to human VEGF. Preclinical studies with a transgenic mouse model of retinal neovascularization showed that the subretinal injection of RGX-314 resulted in significant reduction of neovascularization (89). More recently,
Ding et al. compared the effects of a suprachoroidal and subretinal injection of RGX-314 in rat, and suggested that suprachoroidal delivery of RGX-314 produced comparable anti-VEGF Fab expression and suppression of VEGFinduced hemorrhage (76). A phase I/IIa clinical trial delivering RGX-314 by subretinal injections into nAMD patients is currently ongoing (NCT03066258), and the interim assessment found that RGX-314 was well tolerated and continued to produce anti-VEGF Fab for 2 years $(90,91)$. Interestingly, more than $50 \%$ of patients did not require anti-VEGF injection for as long as 2 years with improved visual acuity and central retinal thickness (90).

\section{RNA interference (RNAi)}

RNAi is a gene silencing mechanism found in eukaryotic cells, in which small interfering RNA (siRNA) guides the cleavage of multiple mRNAs resulting in gene silencing (92). For anti-VEGF therapy, a synthetic siRNA, bevasiranib, suppressed VEGF and laser-induced CNV in human cells lines and mice $(93,94)$. However, the phase III clinical trial (COBALT, NCT00499590) comparing the safety and efficacy of combining bevasiranib with ranibizumab in nAMD patients was terminated due to adverse events including decreased visual acuity, endophthalmitis, uveitis, and cataract formation.

\section{Clustered regularly interspaced short palindromic repeats (CRISPR)-based genome editing}

Despite the early successes of viral-mediated gene therapies, the long-term durability of these treatments remains unclear. Follow-up of Leber congenital amaurosis type 2 patients who received the RPE 65 gene via subretinal AAV2 showed possible loss of efficacy after 2 to 3 years $(95,96)$, although these findings have not been substantiated in later follow-up studies. Nevertheless, new strategies using genome editing have the potential to permanently interrupt pro-angiogenic pathways. CRISPR and CRISPR-associated protein (CRISPR-Cas) systems were discovered as part of bacterial adaptive immunity against viral infection, and are also effective in eukaryotic cells as tools for genome engineering. CRISPR-associated endonucleases such as Cas9 can be programmed using a single guide RNA (gRNA) to target specific sequences in the host genome and create double-strand DNA breaks to create insertions/deletions (indels) of nucleotides that cause frameshift mutations, or enable homology 
directed repair when paired with an additional donor DNA template $(97,98)$. The fast-moving technology has resulted in the rapid discovery of different Cas9 orthologs as well as various editing methods and targets ranging from single base editing to RNA editing that can be utilized in mammalian cells $(99,100)$. While the Cas9 from Streptococcus pyogenes (SpCas9, $4.1 \mathrm{~kb}$ ) has been the best characterized, smaller orthologs from Staphylococcus aureus (SaCas9, $3.1 \mathrm{~kb}$ ) and Campylobacter jejuni (CjCas9, $3 \mathrm{~kb}$ ) have been employed with some success (101). Due to their smaller size, these newer Cas9 variants may have better translational potential when packaged into AAV with gRNAs as "all-in-one" vector systems. The first human clinical trial using subretinal AAV delivery of the CEP290 gene for treatment of Leber congenital amaurosis 10 (LCA10) commenced in late 2019 to determine the safety of this approach (NCT03872479).

Early applications of CRISPR-based genome editing for ocular angiogenesis involved the direct subretinal delivery of CRISPR ribonucleoproteins (RNP) combining both Cas9 endonuclease and gRNA into mouse eyes to ablate the $V E G F$ gene. This approach successfully suppressed laserinduced CNV without significant off-target effects, although the specific cell type targeted was unclear (102). Subsequent studies packaged smaller CRISPR endonucleases such as CjCas9 and Prevoltella and Francisella I (Cpf1, Cas12a) along with respective gRNAs into AAV9 (101,103), and found that targeting either $H I F-1 \alpha$ (the upstream transcriptional regulator of VEGF), or $V E G F a$ resulted in similar levels of CNV suppression when compared with aflibercept, suggesting the potential of CRISPR-based strategies for permanent VEGF suppression and a possible true cure for nAMD (103). In addition, rAAV1 expressing SpCas9 has been used to deplete VEGFR2 in vascular endothelial cells, and successfully suppressed mouse models of oxygeninduced retinopathy (OIR) and laser-induced CNV (104). Efficient suppression of angiogenesis was found despite employing intravitreal delivery of a dual AAV vector system, where Cas9 and the sgRNA were packaged separately.

To evaluate the translational potential of CRISPRbased genome editing for neovascular retinal diseases, our group was the first to demonstrate effective suppression of VEGF secretion from human cells in vitro using a lentiviral vector to express SpCas9 and gRNAs (105). Due to the large size of $\mathrm{SpCas9}$, we subsequently compared subretinal delivery of a dual-AAV vector system to deliver SpCas9 and gRNAs separately, versus a single-AAV vector system to express both SaCas9 and gRNA in mouse eyes in vivo (106). Interestingly, despite similar cutting efficiency in vitro and viral transduction efficiency in vivo between the two platforms, we found that the dual-vector SpCas9 system was more effective in suppressing VEGF than the singlevector SaCas9, suggesting that in vivo performance may be dictated more by the Cas9 ortholog type than the efficiency of viral transduction (106).

Despite early successes in using genome editing to treat ocular angiogenesis, these approaches must be carefully optimized prior to clinical translation. Because genome editing is permanent, and VEGF play physiologic roles in maintaining the health of the retinal and choroidal vasculature, over-suppression may result in harmful adverse effects. Also, the potential for off-target effects as well as the potential for host immune responses due to the exogenous nature of bacterial Cas9 proteins may further pose additional barriers to success. Yet, CRISPR-based strategies may also be particularly suited to address complex, multifactorial processes such as angiogenesis, as it enables multiplexing, where a collection of different gRNAs can be designed to target multiple loci within VEGF or several pro-angiogenic pathways simultaneously (Figure 2). Cellspecific promoters may also be directed to suppress VEGF from more disease-relevant cellular sources, while sparing more physiologic VEGF sources. Thus, future studies to enhance the specificity of CRISPR-based strategies may provide a pathway to enable translation of genome editing to the management of retinal neovascular conditions.

\section{Conclusions}

VEGF plays a significant role in ocular angiogenesis, and anti-VEGF pharmacotherapies have revolutionized the management of neovascular diseases of the retina and enabled the restoration of vision for many patients. However, the frequent need for intraocular injections remain a clinical and financial burden. Recent advances in gene therapy have the potential to improve both the efficacy and durability of anti-VEGF therapies, resolve current unmet needs, and improve patients' quality of life. 
A

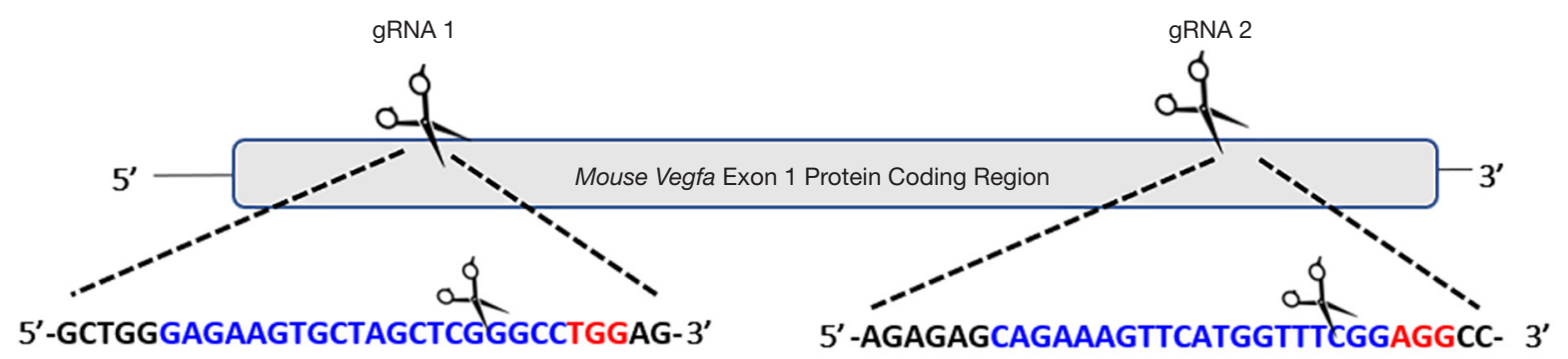

B

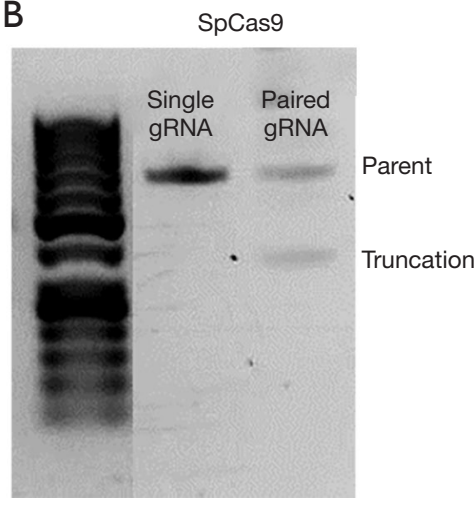

C

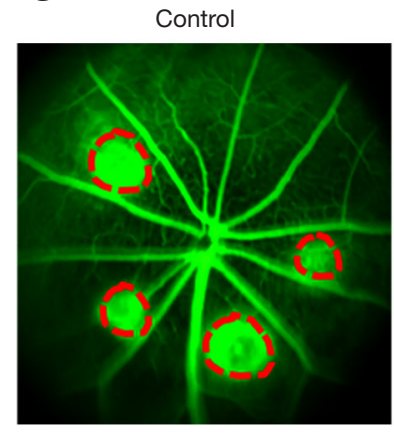

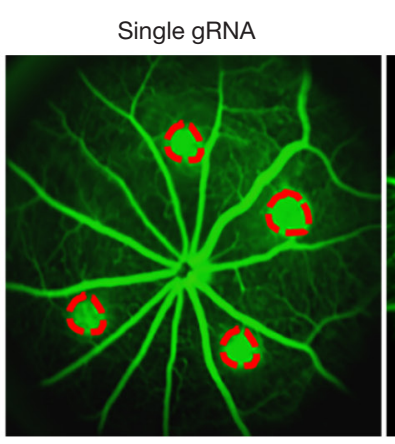

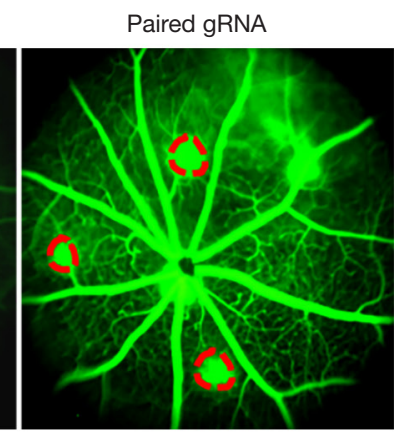

Figure 2 CRISPR technology targeting mouse VEGFa. (A) A schematic diagram of CRISPR genome editing within exon 1 of mouse VEGFa gene. CRISPR with single gRNA targets gRNA1, and CRISPR with paired gRNA system edits both gRNA1 and gRNA2 simultaneously. (B) Genomic analysis showing gene truncation in CRISPR with paired gRNA system. (C) Fluorescence angiography showing suppression of lase-induced CNV after CRISPR delivery in mouse retina. CRISPR, clustered regularly interspaced short palindromic repeats; VEGF, vascular endothelial growth factor; gRNA, guide RNA; CNV, choroidal neovascularization.

\section{Acknowledgments}

Funding: Glenn Yiu is supported by NIH K08 EY026101, NIH R21 EY031108, BrightFocus Foundation, and Macula Society.

\section{Footnote}

Provenance and Peer Review: This article was commissioned by the Guest Editor (Susanna S. Park) for the series "Novel Tools and Therapies for Ocular Regeneration" published in Annals of Translational Medicine. The article has undergone external peer review.

Peer Review File: Available at https://dx.doi.org/10.21037/ atm-20-4417

Conflicts of Interest: All authors have completed the ICMJE uniform disclosure form (available at https://dx.doi. org/10.21037/atm-20-4417). The series "Novel Tools and Therapies for Ocular Regeneration" was commissioned by the editorial office without any funding or sponsorship. GY reports personal fees from Allergan, personal fees and non-financial support from Clearside Biomedical, personal fees from Genentech, grants and personal fees from Iridex, outside the submitted work. The other authors have no other conflicts of interest to declare.

Ethical Statement: The authors are accountable for all aspects of the work in ensuring that questions related to the accuracy or integrity of any part of the work are appropriately investigated and resolved.

Open Access Statement: This is an Open Access article distributed in accordance with the Creative Commons Attribution-NonCommercial-NoDerivs 4.0 International License (CC BY-NC-ND 4.0), which permits the non- 
commercial replication and distribution of the article with the strict proviso that no changes or edits are made and the original work is properly cited (including links to both the formal publication through the relevant DOI and the license). See: https://creativecommons.org/licenses/by-ncnd $/ 4.0 \%$.

\section{References}

1. Penn JS, Madan A, Caldwell RB, et al. Vascular endothelial growth factor in eye disease. Prog Retin Eye Res 2008;27:331-71.

2. Miller JW. VEGF: From Discovery to Therapy: The Champalimaud Award Lecture. Transl Vis Sci Technol 2016;5:9.

3. Ida H, Tobe T, Nambu H, et al. RPE cells modulate subretinal neovascularization, but do not cause regression in mice with sustained expression of VEGF. Invest Ophthalmol Vis Sci 2003;44:5430-7.

4. Miller JW. Vascular endothelial growth factor and ocular neovascularization. Am J Pathol 1997;151:13-23.

5. Robbins SG, Conaway JR, Ford BL, et al. Detection of vascular endothelial growth factor (VEGF) protein in vascular and non-vascular cells of the normal and oxygeninjured rat retina. Growth Factors 1997;14:229-41.

6. Stone J, Chan-Ling T, Pe'er J, et al. Roles of vascular endothelial growth factor and astrocyte degeneration in the genesis of retinopathy of prematurity. Invest Ophthalmol Vis Sci 1996;37:290-9.

7. Aiello LP, Pierce EA, Foley ED, et al. Suppression of retinal neovascularization in vivo by inhibition of vascular endothelial growth factor (VEGF) using soluble VEGFreceptor chimeric proteins. Proc Natl Acad Sci U S A 1995;92:10457-61.

8. Adamis AP, Shima DT, Tolentino MJ, et al. Inhibition of vascular endothelial growth factor prevents retinal ischemia-associated iris neovascularization in a nonhuman primate. Arch Ophthalmol 1996;114:66-71.

9. Tolentino MJ, Miller JW, Gragoudas ES, et al. Vascular endothelial growth factor is sufficient to produce iris neovascularization and neovascular glaucoma in a nonhuman primate. Arch Ophthalmol 1996;114:964-70.

10. Tolentino MJ, Miller JW, Gragoudas ES, et al. Intravitreous injections of vascular endothelial growth factor produce retinal ischemia and microangiopathy in an adult primate. Ophthalmology 1996;103:1820-8.

11. Kazazi-Hyseni F, Beijnen JH, Schellens JHM. Bevacizumab. Oncologist 2010;15:819-25.
12. Michels S, Rosenfeld PJ, Puliafito CA, et al. Systemic bevacizumab (Avastin) therapy for neovascular agerelated macular degeneration twelve-week results of an uncontrolled open-label clinical study. Ophthalmology 2005;112:1035-47.

13. Rosenfeld PJ, Fung AE, Puliafito CA. Optical coherence tomography findings after an intravitreal injection of bevacizumab (avastin) for macular edema from central retinal vein occlusion. Ophthalmic Surg Lasers Imaging 2005;36:336-9.

14. CATT Research Group; Martin DF, Maguire MG, et al. Ranibizumab and bevacizumab for neovascular age-related macular degeneration. N Engl J Med 2011;364:1897-908.

15. Frampton JE. Ranibizumab: A Review of Its Use in the Treatment of Neovascular Age-Related Macular Degeneration. Drugs Aging 2013;30:331-58.

16. Heier JS, Antoszyk AN, Pavan PR, et al. Ranibizumab for treatment of neovascular age-related macular degeneration: a phase I/II multicenter, controlled, multidose study. Ophthalmology 2006;113:633.e1-4.

17. Rosenfeld PJ, Brown DM, Heier JS, et al. Ranibizumab for neovascular age-related macular degeneration. N Engl J Med 2006;355:1419-31.

18. Kaiser PK, Brown DM, Zhang K, et al. Ranibizumab for predominantly classic neovascular age-related macular degeneration: subgroup analysis of first-year ANCHOR results. Am J Ophthalmol 2007;144:850-7.

19. Campochiaro PA, Heier JS, Feiner L, et al. Ranibizumab for macular edema following branch retinal vein occlusion: six-month primary end point results of a phase III study. Ophthalmology 2010;117:1102-1112.e1.

20. Brown DM, Campochiaro PA, Singh RP, et al. Ranibizumab for macular edema following central retinal vein occlusion: six-month primary end point results of a phase III study. Ophthalmology 2010;117:1124-1133.e1.

21. Nguyen QD, Brown DM, Marcus DM, et al. Ranibizumab for diabetic macular edema: results from 2 phase III randomized trials: RISE and RIDE. Ophthalmology 2012;119:789-801.

22. Heier JS, Brown DM, Chong V, et al. Intravitreal Aflibercept (VEGF Trap-Eye) in Wet Age-related Macular Degeneration. Ophthalmology 2012;119:2537-48.

23. Campochiaro PA, Aiello LP, Rosenfeld PJ. Anti-Vascular Endothelial Growth Factor Agents in the Treatment of Retinal Disease. Ophthalmology 2016;123:S78-88.

24. Stewart MW, Rosenfeld PJ, Penha FM, et al. Pharmacokinetic rationale for dosing every 2 weeks versus 4 weeks with intravitreal ranibizumab, bevacizumab, and 
aflibercept (vascular endothelial growth factor Trap-eye).

Retina 2012;32:434-57.

25. Schmidt-Erfurth U, Kaiser PK, Korobelnik JF, et al. Intravitreal Aflibercept Injection for Neovascular Age-related Macular Degeneration. Ophthalmology 2014;121:193-201.

26. Heier JS, Clark WL, Boyer DS, et al. Intravitreal aflibercept injection for macular edema due to central retinal vein occlusion: two-year results from the COPERNICUS study. Ophthalmology 2014;121:14141420.e1.

27. Korobelnik JF, Do DV, Schmidt-Erfurth U, et al. Intravitreal aflibercept for diabetic macular edema. Ophthalmology 2014;121:2247-54.

28. Clark WL, Boyer DS, Heier JS, et al. Intravitreal Aflibercept for Macular Edema Following Branch Retinal Vein Occlusion: 52-Week Results of the VIBRANT Study. Ophthalmology 2016;123:330-6.

29. Korobelnik JF, Holz FG, Roider J, et al. Intravitreal Aflibercept Injection for Macular Edema Resulting from Central Retinal Vein Occlusion: One-Year Results of the Phase 3 GALILEO Study. Ophthalmology 2014;121:202-8.

30. Glassman AR, Wells JA 3rd, Josic K, et al. Five-Year Outcomes after Initial Aflibercept, Bevacizumab, or Ranibizumab Treatment for Diabetic Macular Edema (Protocol T Extension Study). Ophthalmology 2020;127:1201-10.

31. Dugel PU, Koh A, Ogura Y, et al. HAWK and HARRIER: Phase 3, Multicenter, Randomized, Double-Masked Trials of Brolucizumab for Neovascular Age-Related Macular Degeneration. Ophthalmology 2020;127:72-84.

32. Gaudreault J, Gunde T, Floyd HS, et al. Preclinical Pharmacology and Safety of ESBA1008, a Single-chain Antibody Fragment, Investigated as Potential Treatment for Age Related Macular Degeneration. Invest Ophthalmol Vis Sci 2012;53:3025.

33. Tietz J, Spohn G, Schmid G, et al. Affinity and Potency of RTH258 (ESBA1008), a Novel Inhibitor of Vascular Endothelial Growth Factor A for the Treatment of Retinal Disorders. Invest Ophthalmol Vis Sci 2012;56:1501.

34. Holz FG, Dugel PU, Weissgerber G, et al. SingleChain Antibody Fragment VEGF Inhibitor RTH258 for Neovascular Age-Related Macular Degeneration. Ophthalmology 2016;123:1080-9.

35. Dugel PU, Jaffe GJ, Sallstig P, et al. Brolucizumab Versus Aflibercept in Participants with Neovascular AgeRelated Macular Degeneration: A Randomized Trial.
Ophthalmology 2017;124:1296-304.

36. Baumal CR, Spaide RF, Vajzovic L, et al. Retinal Vasculitis and Intraocular Inflammation after Intravitreal Injection of Brolucizumab. Ophthalmology 2020;127:1345-59.

37. Falavarjani KG, Nguyen QD. Adverse events and complications associated with intravitreal injection of anti-VEGF agents: a review of literature. Eye (Lond) 2013;27:787-94.

38. Shah CP, Garg SJ, Vander JF, et al. Outcomes and risk factors associated with endophthalmitis after intravitreal injection of anti-vascular endothelial growth factor agents. Ophthalmology 2011;118:2028-34.

39. Moshfeghi AA, Rosenfeld PJ, Flynn HW Jr, et al. Endophthalmitis after intravitreal vascular [corrected] endothelial growth factor antagonists: a six-year experience at a university referral center. Retina 2011;31:662-8. Erratum in: Retina. 2012 Jul;32(7):1441.

40. Campochiaro PA, Marcus DM, Awh CC, et al. The Port Delivery System with Ranibizumab for Neovascular Age-Related Macular Degeneration. Ophthalmology 2019;126:1141-54.

41. Rodrigues GA, Mason M, Christie LA, et al. Functional Characterization of Abicipar-Pegol, an Anti-VEGF DARPin Therapeutic That Potently Inhibits Angiogenesis and Vascular Permeability. Invest Ophthalmol Vis Sci 2018;59:5836.

42. Callanan D, Kunimoto D, Maturi RK, et al. DoubleMasked, Randomized, Phase 2 Evaluation of Abicipar Pegol (an Anti-VEGF DARPin Therapeutic) in Neovascular Age-Related Macular Degeneration. J Ocul Pharmacol Ther 2018;34:700-9.

43. Kunimoto D, Yoon YH, Wykoff CC, et al. Efficacy and Safety of Abicipar in Neovascular Age-Related Macular Degeneration: 52-Week Results of Phase 3 Randomized Controlled Study. Ophthalmology 2020;127:1331-44.

44. Wang CH, Hwang YS, Chiang PR, et al. Extended Release of Bevacizumab by Thermosensitive Biodegradable and Biocompatible Hydrogel. Biomacromolecules. 2012;13:40-8.

45. Liu W, Borrell MA, Venerus DC, et al. Characterization of Biodegradable Microsphere-Hydrogel Ocular Drug Delivery System for Controlled and Extended Release of Ranibizumab. Transl Vis Sci Technol 2019;8:12.

46. Liu W, Lee BS, Mieler WF, et al. Biodegradable Microsphere-Hydrogel Ocular Drug Delivery System for Controlled and Extended Release of Bioactive Aflibercept In Vitro. Curr Eye Res 2019;44:264-74.

47. Kim S, Kang-Mieler JJ, Liu W, et al. Safety and 
Biocompatibility of Aflibercept-Loaded Microsphere Thermo-Responsive Hydrogel Drug Delivery System in a Nonhuman Primate Model. Transl Vis Sci Technol 2020;9:30.

48. Boyer DS, Yoon YH, Belfort R, et al. Three-year, randomized, sham-controlled trial of dexamethasone intravitreal implant in patients with diabetic macular edema. Ophthalmology 2014;121:1904-14.

49. Callanan DG, Gupta S, Boyer DS, et al. Dexamethasone intravitreal implant in combination with laser photocoagulation for the treatment of diffuse diabetic macular edema. Ophthalmology 2013;120:1843-51.

50. Haller JA, Bandello F, Belfort R, et al. Randomized, shamcontrolled trial of dexamethasone intravitreal implant in patients with macular edema due to retinal vein occlusion. Ophthalmology 2010;117:1134-1146.e3.

51. Luis de Redín I, Boiero C, Recalde S, et al. In vivo effect of bevacizumab-loaded albumin nanoparticles in the treatment of corneal neovascularization. Exp Eye Res 2019;185:107697.

52. Moisseiev E, Loewenstein A. Abicipar pegol-a novel anti-VEGF therapy with a long duration of action. Eye 2020;34:605-6.

53. Plückthun A. Designed Ankyrin Repeat Proteins (DARPins): Binding Proteins for Research, Diagnostics, and Therapy. Annu Rev Pharmacol Toxicol 2015;55:489-511.

54. Campochiaro PA, Channa R, Berger BB, et al. Treatment of diabetic macular edema with a designed ankyrin repeat protein that binds vascular endothelial growth factor: a phase I/II study. Am J Ophthalmol 2013;155:697-704, 704.e1-2.

55. Krohne TU, Liu Z, Holz FG, et al. Intraocular Pharmacokinetics of Ranibizumab Following a Single Intravitreal Injection in Humans. American Journal of Ophthalmology 2012;154:682-686.e2.

56. Li J, Mooney D. Designing hydrogels for controlled drug delivery. Nat Rev Mater 2016;1:16071.

57. Wichterle O, Lím D. Hydrophilic Gels for Biological Use. Nature 1960;185:117-8.

58. Kachi S, Binley K, Yokoi K, et al. Equine infectious anemia viral vector-mediated codelivery of endostatin and angiostatin driven by retinal pigmented epithelium-specific VMD2 promoter inhibits choroidal neovascularization. Hum Gene Ther 2009;20:31-9.

59. Matanović MR, Kristl J, Grabnar PA. Thermoresponsive polymers: insights into decisive hydrogel characteristics, mechanisms of gelation, and promising biomedical applications. Int J Pharm 2014;472:262-75

60. Shoval A, Markus A, Zhou Z, et al. Anti-VEGFAptamer Modified C-Dots-A Hybrid Nanocomposite for Topical Treatment of Ocular Vascular Disorders. Small 2019;15:e1902776.

61. Miraldi Utz V, Coussa RG, Antaki F, et al. Gene therapy for RPE65-related retinal disease. Ophthalmic Genet 2018;39:671-7.

62. Cideciyan AV. Leber congenital amaurosis due to RPE65 mutations and its treatment with gene therapy. Prog Retin Eye Res 2010;29:398-427.

63. Makadia HK, Siegel SJ. Poly Lactic-co-Glycolic Acid (PLGA) as Biodegradable Controlled Drug Delivery Carrier. Polymers 2011;3:1377-97.

64. Yáñez-Muñoz RJ, Balaggan KS, MacNeil A, et al. Effective gene therapy with nonintegrating lentiviral vectors. Nat Med 2006;12:348-53.

65. Wang D, Tai PWL, Gao G. Adeno-associated virus vector as a platform for gene therapy delivery. Nat Rev Drug Discov 2019;18:358-78.

66. Lotery AJ, Yang GS, Mullins RF, et al. Adenoassociated virus type 5: transduction efficiency and celltype specificity in the primate retina. Hum Gene Ther 2003;14:1663-71.

67. Burnight ER, Giacalone JC, Cooke JA, et al. CRISPRCas9 genome engineering: Treating inherited retinal degeneration. Prog Retin Eye Res 2018;65:28-49.

68. Auricchio A, Kobinger G, Anand V, et al. Exchange of surface proteins impacts on viral vector cellular specificity and transduction characteristics: the retina as a model. Hum Mol Genet 2001;10:3075-81.

69. Dalkara D, Byrne LC, Klimczak RR, et al. In vivo-directed evolution of a new adeno-associated virus for therapeutic outer retinal gene delivery from the vitreous. Sci Transl Med 2013;5:189ra76.

70. Takahashi K, Igarashi T, Miyake K, et al. Improved Intravitreal AAV-Mediated Inner Retinal Gene Transduction after Surgical Internal Limiting Membrane Peeling in Cynomolgus Monkeys. Mol Ther 2017;25:296-302.

71. Emami-Naeini P, Yiu G. Medical and Surgical Applications for the Suprachoroidal Space. Int Ophthalmol Clin 2019;59:195-207.

72. Yiu G, Pecen P, Sarin N, et al. Characterization of the choroid-scleral junction and suprachoroidal layer in healthy individuals on enhanced-depth imaging optical coherence tomography. JAMA Ophthalmol 2014;132:174-81. 
73. Moisseiev E, Loewenstein A, Yiu G. The suprachoroidal space: from potential space to a space with potential. Clin Ophthalmol 2016;10:173-8.

74. Yeh S, Khurana RN, Shah M, et al. Efficacy and Safety of Suprachoroidal CLS-TA for Macular Edema Secondary to Noninfectious Uveitis: Phase 3 Randomized Trial. Ophthalmology 2020;127:948-55.

75. Willoughby AS, Vuong VS, Cunefare D, et al. Choroidal Changes After Suprachoroidal Injection of Triamcinolone Acetonide in Eyes With Macular Edema Secondary to Retinal Vein Occlusion. Am J Ophthalmol 2018;186:144-51.

76. Ding K, Shen J, Hafiz Z, et al. AAV8-vectored suprachoroidal gene transfer produces widespread ocular transgene expression. J Clin Invest 2019;129:4901-11.

77. Yiu G, Chung SH, Mollhoff IN, et al. Suprachoroidal and Subretinal Injections of AAV Using Transscleral Microneedles for Retinal Gene Delivery in Nonhuman Primates. Mol Ther Methods Clin Dev 2020;16:179-91.

78. Peden MC, Min J, Meyers C, et al. Ab-externo AAVmediated gene delivery to the suprachoroidal space using a 250 micron flexible microcatheter. PLoS One 2011;6:e17140.

79. Martorana G, Levine M, Peden M, et al. Comparison of Suprachoroidal delivery via an Ab-Externo approach with the iTrack Microcatheter versus Vitrectomy and subretinal delivery for 3 different AAV Serotypes for Gene Transfer to the Retina. Invest Ophthalmol Vis Sci 2012;53:1931.

80. Lai CM, Shen WY, Brankov M, et al. Long-term evaluation of AAV-mediated sFlt-1 gene therapy for ocular neovascularization in mice and monkeys. Mol Ther 2005;12:659-68.

81. Lai CM, Estcourt MJ, Wikstrom M, et al. rAAV.sFlt1 Gene Therapy Achieves Lasting Reversal of Retinal Neovascularization in the Absence of a Strong Immune Response to the Viral Vector. Invest Ophthalmol Vis Sci 2009;50:4279.

82. Lai CM, Estcourt MJ, Himbeck RP, et al. Preclinical safety evaluation of subretinal AAV2.sFlt-1 in non-human primates. Gene Ther 2012;19:999-1009.

83. Rakoczy EP, Lai CM, Magno AL, et al. Gene therapy with recombinant adeno-associated vectors for neovascular agerelated macular degeneration: 1 year follow-up of a phase 1 randomised clinical trial. Lancet 2015;386:2395-403.

84. Constable IJ, Pierce CM, Lai CM, et al. Phase 2a Randomized Clinical Trial: Safety and Post Hoc Analysis of Subretinal rAAV.sFLT-1 for Wet Age-related Macular Degeneration. EBioMedicine 2016;14:168-75.
85. Heier JS, Kherani S, Desai S, et al. Intravitreous injection of AAV2-sFLT01 in patients with advanced neovascular age-related macular degeneration: a phase 1, open-label trial. Lancet 2017;390:50-61.

86. Grishanin R, Vuillemenot B, Sharma P, et al. Preclinical Evaluation of ADVM-022, a Novel Gene Therapy Approach to Treating Wet Age-Related Macular Degeneration. Mol Ther 2019;27:118-29.

87. Khanani AM, Kiss S, Turpcu A, et al. Phase 1 Study of Intravitreal Gene Therapy ADVM-022 for neovascular AMD (OPTIC Trial). Invest Ophthalmol Vis Sci 2020;61:1154.

88. Khanani AM, Kiss S, Turpcu A, et al. Phase 1 Study of Intravitreal Gene Therapy ADVM-022 for neovascular AMD (OPTIC Trial). Invest Ophthalmol Vis Sci 2020;61:1154.

89. Liu Y, Fortmann SD, Shen J, et al. AAV8-antiVEGFfab Ocular Gene Transfer for Neovascular Age-Related Macular Degeneration. Mol Ther 2018;26:542-9.

90. REGENXBIO Reports Continued Progress Across Programs in Year-End 2018 Corporate Update I REGENXBIO Inc. [Internet]. [cited 2020 May 16]. Available online: https://regenxbio.gcs-web.com/ news-releases/news-release-details/regenxbio-reportscontinued-progress-across-programs-year-end

91. REGENXBIO Announces Additional Positive Long-term and Interim Phase I/IIa Trial Update for RGX-314 for the Treatment of Wet AMD I REGENXBIO Inc. [Internet]. [cited 2020 May 16]. Available online: https://regenxbio. gcs-web.com/news-releases/news-release-details/ regenxbio-announces-additional-positive-long-term-andinterim

92. Dykxhoorn DM, Palliser D, Lieberman J. The silent treatment: siRNAs as small molecule drugs. Gene Ther 2006;13:541-52.

93. Xia XB, Xiong S, Song W, et al. Inhibition of retinal neovascularization by siRNA targeting $\operatorname{VEGF}(165)$. Mol Vis 2008;14:1965-73.

94. Reich SJ, Fosnot J, Kuroki A, et al. Small interfering RNA (siRNA) targeting VEGF effectively inhibits ocular neovascularization in a mouse model. Mol Vis 2003;9:210-6.

95. Jacobson SG, Cideciyan AV, Roman AJ, et al. Improvement and Decline in Vision with Gene Therapy in Childhood Blindness. N Engl J Med 2015;372:1920-6.

96. Bainbridge JWB, Mehat MS, Sundaram V, et al. LongTerm Effect of Gene Therapy on Leber's Congenital Amaurosis. N Engl J Med 2015;372:1887-97. 
97. DiCarlo JE, Sengillo JD, Justus S, et al. CRISPR-Cas Genome Surgery in Ophthalmology. Transl Vis Sci Technol 2017;6:13.

98. Yiu G. Genome Editing in Retinal Diseases using CRISPR Technology. Ophthalmol Retina 2018;2:1-3. Erratum in: Ophthalmol Retina. 2018 May;2(5):514.

99. Cox DBT, Gootenberg JS, Abudayyeh OO, et al. RNA editing with CRISPR-Cas13. Science 2017;358:1019-27.

100. Kim K, Ryu SM, Kim ST, et al. Highly efficient RNAguided base editing in mouse embryos. Nat Biotechnol 2017;35:435-7.

101. Kim E, Koo T, Park SW, et al. In vivo genome editing with a small Cas9 orthologue derived from Campylobacter jejuni. Nat Commun 2017;8:14500.

102. Kim K, Park SW, Kim JH, et al. Genome surgery using Cas9 ribonucleoproteins for the treatment of age-related

Cite this article as: Chung SH, Frick SL, Yiu G. Targeting vascular endothelial growth factor using retinal gene therapy. Ann Transl Med 2021;9(15):1277. doi: 10.21037/atm-20-4417 macular degeneration. Genome Res 2017;27:419-26.

103. Koo T, Park SW, Jo DH, et al. CRISPR-LbCpf1 prevents choroidal neovascularization in a mouse model of agerelated macular degeneration. Nat Commun 2018;9:1855.

104.Huang X, Zhou G, Wu W, et al. Genome editing abrogates angiogenesis in vivo. Nat Commun 2017;8:112.

105. Yiu G, Tieu E, Nguyen AT, et al. Genomic Disruption of VEGF-A Expression in Human Retinal Pigment Epithelial Cells Using CRISPR-Cas9 Endonuclease. Invest Ophthalmol Vis Sci 2016;57:5490.

106. Chung SH, Mollhoff IN, Nguyen U, et al. Factors Impacting Efficacy of AAV-Mediated CRISPRBased Genome Editing for Treatment of Choroidal Neovascularization. Mol Ther Methods Clin Dev 2020;17:409-17. 